\title{
Avaliação da ansiedade em indivíduos submetidos a exodontia de terceiros molares inferiores em um ensaio clínico do tipo split-mouth, triplo cego randomizado
}

Anxiety assessment in individuals undergoing lower third molar extraction in a split-mouth, triple blind randomized clinical trial

Evaluación de la ansiedad en individuos sometidos a extracción del tercer molar inferior en un ensayo clínico aleatorizado triple ciego de boca dividida

\author{
Renata Lanzoni de Oliveira \\ ORCID: https://orcid.org/0000-0002-1124-5324 \\ Universidade Federal de Mato Grosso do Sul, Brasil \\ E-mail: re_lanzoni@hotmail.com \\ Paulo Roberto Haidamus de Oliveira Bastos \\ ORCID: https://orcid.org/0000-0002-8885-1461 \\ Universidade Federal de Mato Grosso do Sul, Brasil \\ E-mail: phaidamus43@gmail.com \\ Janayna Gomes Paiva Oliveira \\ ORCID: https://orcid.org/0000-0003-2090-2872 \\ Universidade Federal do Mato Grosso do Sul, Brasil \\ E-mail:janaynajgpaivaoliveira@gmail.com \\ Ellen Cristina Gaetti Jardim \\ ORCID: https://orcid.org/0000-0003-2471-465X \\ Universidade Federal do Mato Grosso do Sul, Brasil \\ E-mail: ellen.jardim@ufms.br \\ Nathani Hevelin Moreira de Araujo \\ ORCID: https://orcid.org/0000-0001-7601-5477 \\ Universidade Federal de Mato Grosso do Sul, Brasil \\ E-mail: nathani_araujo23@hotmail.com \\ Paulo Pereira do Nascimento \\ ORCID: https://orcid.org/0000-0001-7688-8833 \\ Universidade Federal de Mato Grosso do Sul, Brasil \\ E-mail: paulo_pnascimento@hotmail.com \\ Francielly Thomas Figueiredo \\ ORCID: https://orcid.org/0000-0003-0196-1172 \\ Universidade Federal do Mato Grosso do Sul, Brasil \\ E-mail: francielly.thomas@ufms.br \\ Gustavo Silva Pelissaro \\ ORCID: https://orcid.org/0000-0003-3475-6001 \\ Universidade Federal do Mato Grosso do Sul, Brasil \\ E-mail: gustavopelissaro@hotmail.com \\ Victor Greve Goes \\ ORCID: https://orcid.org/0000-0001-5267-2296 \\ Médico, Brasil \\ E-mail: victor.greve@gmail.com \\ Camila Stevaneli Freitas Grande \\ ORCID: https://orcid.org/0000-0002-3452-4182 \\ Cirurgiã-dentista, Brasil \\ E-mail: camilastevaneli@gmail.com
}

\begin{abstract}
Resumo
Para muitos indivíduos o ambiente odontológico remete a uma situação de medo e ansiedade. Para que seja estabelecido um ambiente favorável ao profissional e paciente, o controle da ansiedade é de fundamental importância. Este ensaio clínico, prospectivo, do tipo split-mouth, triplo-cego e randomizado teve por objetivo avaliar a ansiedade em pacientes que seriam submetidos a exodontia de terceiros molares inferiores com sedação consciente mínima utilizando o extrato fixo de Valeriana-Lúpulo e o Midazolam. Concluíram a pesquisa, 12 indivíduos com idade entre 18 e 25 anos, de ambos os gêneros, com classificação de risco cirúrgico ASA I. Para avaliar a ansiedade foi utilizada em três momentos a Escala de Ansiedade Odontológica de Corah Modificada. Os participantes receberam por via oral, 1 cápsula de Valeriana 500 mg - Lúpulo $120 \mathrm{mg}$ ou Midazolam $15 \mathrm{mg}$ para cada lado a ser operado, 45 minutos antes do início do procedimento cirúrgico. Não houve diferenças nos escores de ansiedade entre a $1^{\mathrm{a}}$ e $2^{\mathrm{a}}$ cirurgias para ambos os tratamentos. Foi
\end{abstract}


possível ratificar a eficácia do Midazolam para realização de sedação consciente mínima, pois os resultados se apresentaram melhores que a Valeriana-Lúpulo sobre a variável abordada, contudo, há a necessidade da elaboração de mais estudos acerca dos efeitos da associação Valeriana-Lúpulo para controle da ansiedade odontológica.

Palavras-chave: Ansiedade; Plantas medicinais; Medicamentos fitoterápicos.

\begin{abstract}
For many individuals, the dental environment refers to a situation of fear and anxiety. In order to establish a favorable environment for professionals and patients, anxiety control is of fundamental importance. This prospective, split-mouth, triple-blind, randomized clinical trial aimed to assess anxiety in patients who would undergo lower third molar extraction with minimal conscious sedation using Valerian-Hop fixed extract and Midazolam. Twelve individuals aged between 18 and 25 years, of both genders, with surgical risk classification ASA I concluded the research. To assess anxiety, the Modified Corah Dental Anxiety Scale was used in three moments. Participants received, orally, 1 capsule of Valeriana $500 \mathrm{mg}$ - Hops $120 \mathrm{mg}$ or Midazolam $15 \mathrm{mg}$ for each side to be operated, 45 minutes before the beginning of the surgical procedure. There were no differences in anxiety scores between the 1st and 2nd surgeries for both treatments. It was possible to confirm the effectiveness of Midazolam to perform minimal conscious sedation, as the results were better than Valerian-Hop on the variable addressed, however, there is a need for further studies on the effects of the Valerian-Hop association for control of dental anxiety.
\end{abstract}

Keywords: Anxiety; Plants, medicinal; Phytotherapeutic drugs.

\title{
Resumen
}

Para muchas personas, el entorno dental se refiere a una situación de miedo y ansiedad. Para establecer un entorno favorable para los profesionales y los pacientes, el control de la ansiedad es de fundamental importancia. Este ensayo clínico prospectivo, de boca dividida, triple ciego y aleatorizado tuvo como objetivo evaluar la ansiedad en pacientes que se someterían a la extracción del tercer molar inferior con una mínima sedación consciente utilizando extracto fijo de Valerian-Hop y Midazolam. Doce individuos de entre 18 y 25 años, de ambos sexos, con clasificación de riesgo quirúrgico ASA I. Para evaluar la ansiedad se utilizó la Escala de Ansiedad Dental Corah Modificada en tres momentos. Los participantes recibieron, por vía oral, 1 cápsula de Valeriana $500 \mathrm{mg}$ - Lúpulo $120 \mathrm{mg}$ o Midazolam $15 \mathrm{mg}$ por cada lado a operar, 45 minutos antes del inicio del procedimiento quirúrgico. No hubo diferencias en las puntuaciones de ansiedad entre la $1^{\mathrm{a}}$ y la $2^{\mathrm{a}}$ cirugías para ambos tratamientos. Se pudo confirmar la efectividad del Midazolam para realizar sedación mínima consciente, ya que los resultados fueron mejores que Valerian-Hop en la variable abordada, sin embargo, se necesitan más estudios sobre los efectos de la asociación Valerian-Hop para el control de ansiedad dental.

Palabras clave: Ansiedad; Plantas medicinales; Medicamentos fitoterápicos.

\section{Introdução}

Para muitos indivíduos o ambiente odontológico remete a uma situação de medo e ansiedade (Possobon et al., 2007;

Farah, 2010). Estes sentimentos são multifatoriais e repercutem diretamente na condição psicológica e fisiológica do ser humano (Dhuvad et al., 2014).

Sinais clínicos de ansiedade podem ser observados por meio de manifestações corporais involuntárias como palidez, transpiração excessiva, tremores, tonturas, dilatação da pupila, boca seca, dificuldade respiratória (Dantas et al., 2017), aumento da sensação de dor (Van Wijk, Hoogstraten, 2005; Guzeldemir et al., 2008; Sanikop et al., 2011), além de alterações hemodinâmicas sobre a pressão arterial, frequência cardíaca, respiratória e saturação do oxigênio (Lago-Mendez et al., 2006; Braga et al., 2010; Joshi et al., 2016).

A forma mais frequente de mensurar a ansiedade é por meio da Escala de Ansiedade Odontológica, desenvolvida por Corah, em 1978. Este teste, de acordo com a literatura mais recente, é simples e considerado o melhor em termos de consistência interna e confiabilidade teste - reteste (Armfield \& Heaton, 2013).

A sedação consciente mínima com benzodiazepínicos é a mais comum e eficiente alternativa para controle da ansiedade no consultório odontológico, por promover mínima depressão do nível de consciência, sem interferir em sua capacidade respiratória independente, na percepção a estímulos físicos e ao comando verbal (Donaldson \& Chanpong, 2007). A função cognitiva e coordenação motora são pouco alteradas (ADA 1999; ADA 2007), podendo promover também analgesia e amnésia anterógrada (Ong et al., 2005).

Porém, podem causar efeitos secundários, tais como prurido, dor de cabeça, náusea, aumento do efeito do álcool, efeito 
paradoxal (Dantas et al., 2017) e depressão respiratória e/ou uma dessaturação de oxigênio (ADA, 1999). Por este motivo, devem sempre estar acompanhados nas consultas (Cogo et al., 2006).

Com a finalidade de reduzir ou evitar os efeitos secundários dos benzodiazepínicos, fármacos fitoterápicos têm sido administrados para controlar a ansiedade, tais como extrato de Valeriana (Pinheiro et al., 2014). A fitoterapia vem sendo largamente empregada nas patologias psiquiátricas como depressão e ansiedade, por apresentarem menores efeitos colaterais e risco de dependência química, além de ter um menor custo que os benzodiazepínicos (Kinrys et al., 2009).

Diante disso, o presente estudo justifica-se pela necessidade de novas alternativas de fármacos que possam ajudar na sedação consciente do paciente sem que haja efeitos colaterais.

Como relevância científica o presente estudo ajudará os profissionais a prescreverem fármacos fitoterápicos de forma segura e eficiente.

\section{Metodologia}

Trata-se de um ensaio clínico prospectivo do tipo split-mouth (boca dividida), triplo-cego, randomizado. Este estudo foi submetido à análise do Comitê de Ética em Pesquisa da Universidade Federal de Mato Grosso do Sul em dois momentos e aprovado sob os pareceres 64575317.2.0000.0021 e 4.908.740.

\section{Desenho experimental}

Fizeram parte deste estudo doze participantes com classificação de risco cirúrgico ASA I (American Society of Anesthesiologists) com faixa etária entre 18 e 25 anos, de ambos os sexos, com indicação ortodôntica para exodontia dos terceiros molares inferiores retidos.

O estudo foi conduzido de acordo com os Guidelines de CONSORT (Consolidated Standards of Reporting Trials) para ensaios clínicos.

Como critério de inclusão: para participar da pesquisa os voluntários selecionados deviam ser considerados ansiosos e possuir terceiros molares inferiores (38 e 48) retidos bilateralmente tendo a mesma classificação de Pell e Gregory e Miller Winter, tais elementos deveriam necessitar da utilização de instrumento rotatório para sua remoção tanto para ostectomia e/ou odontossecção.

Como critério de exclusão: participantes que não aceitassem assinar o TCLE, participantes com pericoronarite prévia, alérgicos aos fármacos e materiais utilizados no estudo, tabagistas, gestantes e lactantes, indivíduos que fizessem uso de medicação contínua (incluindo anticoncepcionais) ou que tivessem feito uso de medicamentos anti-inflamatórios e analgésicos até 15 dias do início de sua participação no estudo, indivíduos que não pudessem retornar para segunda sessão cirúrgica, que fizessem uso de outro tipo de medicação que não as fornecidas pelo estudo, participantes que não respondessem aos questionários da pesquisa, aqueles que se mostrassem não colaborativos aos protocolos farmacológicos instituídos e por fim aqueles que desenvolvessem algum tipo de infecção local no pós-operatório.

\section{Orientações pré e pós-operatórias}

Os participantes da pesquisa foram orientados na consulta inicial quanto ao tipo de alimentação que deveriam fazer nas horas anteriores ao procedimento cirúrgico e no pós-operatório. Também foram orientados a não consumir álcool 24 horas antes e após o procedimento, e não dirigir, operar máquinas ou equipamentos que exijam atenção nas primeiras 12 horas pósoperatórias. Foi orientado que no dia do procedimento cirúrgico deveriam chegar pelo menos 1 hora e 30 minutos antes do início da cirurgia, devidamente acompanhados por um responsável maior de 18 anos de idade. 


\section{Randomização e mascaramento}

Os participantes foram distribuídos de maneira randomizada em dois grupos de sedação. Os doze receberam de um dos lados a serem operados o tratamento (1A), onde foi administrado por via oral Valeriana $500 \mathrm{mg}$ - Lúpulo $120 \mathrm{mg}$, $45 \mathrm{minutos}$ antes de começar a cirurgia. Já para o outro lado os mesmos indivíduos receberam o tratamento (1B), onde foi administrado por via oral, Midazolam 15 mg, 45 minutos antes do início da cirurgia.

Com o objetivo de garantir que o participante e o pesquisador principal não soubessem qual fármaco estava sendo administrado antes do procedimento, as cápsulas de Valeriana $500 \mathrm{mg}$ - Lúpulo $120 \mathrm{mg}$ e Midazolam $15 \mathrm{mg}$ foram manipuladas e encapsuladas de forma idêntica em sua aparência (Farmácia de Manipulação Tavepharma - São Paulo - SP, Brasil), sendo separadas por um pesquisador auxiliar em dois frascos denominados tratamento $1 \mathrm{~A}$ e tratamento $1 \mathrm{~B}$. Com finalidade de promover analgesia preemptiva e controlar o processo inflamatório no pós-operatório foi também administrado 1 cápsula de dexametasona $8 \mathrm{mg}$ (Farmácia de Manipulação Tavepharma - São Paulo - SP, Brasil) 1 hora antes do procedimento cirúrgico por via oral para ambos os lados a serem operados. Os códigos utilizados na randomização dos tratamentos (1A e 1B) só foram revelados após análise de todos os dados dos participantes envolvidos com a pesquisa.

Os doze indivíduos selecionados foram alocados por randomização simples, de forma alternada e determinada previamente a sorte, que os participantes de número ímpar teriam o tratamento (1A ou 1B) e lado a ser operado (direito ou esquerdo) sorteados na primeira cirurgia. O participante subsequente, o de número par, teve em sua primeira cirurgia, o tratamento e lado oposto ao sorteado para primeira cirurgia do participante de número ímpar e assim foi feito sucessivamente, sempre com novos sorteios, tanto dos tratamentos quanto dos lados para os participantes ímpares em suas primeiras cirurgias e consequentemente, a eleição dos tratamentos e lados opostos para as primeiras cirurgias para os participantes de número par.

De forma que, cada participante teve os tratamentos sedativos 1A e 1B e os lados a serem operados (direito e esquerdo) com a mesma oportunidade de ser a primeira e a segunda sessão cirúrgica. O lado a ser operado foi informado pelo pesquisador auxiliar ao cirurgião e pesquisador principal. Desta forma, o cirurgião e o participante foram mascarados quanto ao tipo de fármaco sedativo utilizado em cada procedimento cirúrgico.

\section{Procedimentos cirúrgicos e medicações}

As intervenções cirúrgicas foram realizadas por um único especialista em cirurgia e traumatologia buco-maxilo-facial, em duas sessões diferentes, com um intervalo de 21 dias entre elas, para que o processo inflamatório e de cicatrização da primeira cirurgia não interferisse na percepção da dor do lado contralateral. Em cada cirurgia o participante recebeu um tipo de sedativo conforme a randomização. Além disso, uma hora antes do início do procedimento cirúrgico os indivíduos receberam dexametasona $8 \mathrm{mg}$ por via oral. Os voluntários da pesquisa foram submetidos à antissepsia intra-bucal com solução aquosa de digluconato de clorexidina a 0,12\% (Farmácia de Manipulação São Bento - Brasil) por meio de bochecho vigoroso de $10 \mathrm{ml}$ da solução por 1 minuto, e antissepsia extraoral com solução tópica de clorexidina a 2\% (Farmácia de Manipulação São Bento Brasil). Foi realizada inicialmente uma anestesia tópica com Lidocaína a 10\% (Xylocaína Spray 10\%, AstraZeneca - Brasil). A anestesia local foi realizada com Articaína 4\% com epinefrina 1:100.000 (Articaine, DFL - Brasil), com um volume máximo de até 4,5 ml, equivalente ao contido em $(2,5)$ dois tubetes anestésico e meio. Foi realizada uma incisão sulcular, com lâmina de bisturi número 15C (Med Blade - Brasil), na região de crista óssea do terceiro molar inferior e na região do segundo molar inferior. Na sequência, foi feito o descolamento mucoperiostal, osteotomia e odontossecção. A remoção dental foi concluída com extratores curvos e retos do tipo Seldin (n 2, 1R ou 1L), em seguida, foi feita uma inspeção cuidadosa para remoção do folículo pericoronário utilizando cureta de Lucas e pinça Kelly curva (Quinelato - Brasil). As bordas ósseas da loja cirúrgica foram regularizadas com lima para osso (Quinelato - Brasil) para remoção das esquírulas ósseas e, em seguida foi realizada uma irrigação abundante com soro fisiológico $\mathrm{NaCl}$ 0,9\% estéril (Sanobiol - Brasil). Após o preenchimento da loja cirúrgica por 
sangue, realizou-se suturas interrompidas com fio de seda trançada 4.0 (Ethicon, Jhonson \& Jhonson - Brasil). Devido à possibilidade de algum grau de depressão respiratória causada pelos fármacos sedativos, o oxímetro de pulso digital foi mantido para o monitoramento antes, durante e depois da cirurgia a fim de detectar alterações.

\section{Avaliação da ansiedade}

A ansiedade foi avaliada na consulta inicial de seleção dos participantes e no pré-operatório (1 hora antes do início do procedimento cirúrgico) na primeira e na segunda cirurgia, segundo a Escala de Ansiedade Odontológica de Corah Modificada (MDAS), descrita por Corah, modificada por HUMPHRIS.

Foi fornecido ao participante uma ficha impressa contendo cinco perguntas objetivas e através do somatório das respostas foi possível classificar a ansiedade de cada participante em: pouco (até 5 pontos), levemente (6 a 10 pontos), moderadamente (11 a 15 pontos) e extremamente ansioso (16 a 25 pontos).

Quadro 1 - Classificação de ansiedade, segundo MDAS, traduzido.

\begin{tabular}{|c|c|}
\hline Até 5 pontos & Pouco ansioso (baixa ansiedade) \\
\hline 6 a 10 pontos & Levemente ansioso (baixa ansiedade) \\
\hline 11 a 15 pontos & Moderadamente ansioso (ansiedade moderada) \\
\hline 16 a 25 pontos & Extremamente ansioso (alta ansiedade) \\
\hline
\end{tabular}

Fonte: Corah et al. (1978). Assessment of a dental anxiety scale. The Journal of the American Dental Association. 97, 816-19.

\section{Avaliação do nível de sedação}

Para avaliar o nível de sedação do paciente foi utilizado no estudo a Escala de Ramsay sendo aplicada 45 minutos após a administração do agente sedativo por via oral. A escala possui valores (de 1 a 6) atribuídos pelo avaliador sobre a sedação alcançada pelo paciente, observando as respostas produzidas pelo paciente após a sedação, de acordo com o julgamento do operador, sendo: grau 1 - paciente ansioso, agitado; grau 2 - cooperativo, orientado, tranquilo; grau 3 - sonolento, atendendo aos comandos; grau 4 - dormindo, responde rapidamente ao estímulo glabelar ou ao estímulo sonoro vigoroso; grau 5 dormindo, responde lentamente ao estímulo glabelar ou ao estímulo sonoro vigoroso e grau 6 - dormindo, sem resposta (Quadro 2).

Quadro 2 - Grau de ansiedade, segundo escala de Ramsay, traduzido.

\begin{tabular}{|c|c|}
\hline Grau 1 & Paciente ansioso, agitado \\
\hline Grau 2 & Cooperativo, orientado, tranquilo \\
\hline Grau 3 & Sonolento, atento aos comandos \\
\hline Grau 4 & $\begin{array}{c}\text { Dormindo, responde rapidamente ao estímulo glabelar ou ao estímulo } \\
\text { sonoro vigoroso }\end{array}$ \\
\hline Grau 5 & Dormindo, responde lentamente ao estímulo glabelar ou ao estímulo \\
& sonoro vigoroso \\
\hline Grau 6 & Dormindo, sem resposta \\
\hline
\end{tabular}

Fonte: Ramsay, et al. (1974). Controlled sedation with alphaxalone-alphadolone. British Medical Journal. 2(5920), 656. 
Para realização da sedação consciente mínima foi utilizado os Guidelines da ADA 2012, tendo o cirurgião principal certificação em Suporte Básico de Vida, estando disponível dentre os materiais e equipamentos para realização do mesmo, tais como oxigênio, se porventura houvesse dessaturação e o fármaco Flumazenil para realização de manobras de reversão do efeito do Midazolam por equipe médica, se necessário. A pesquisa foi realizada em ambiente ambulatorial da clínica de Cirurgia e Traumatologia Bucomaxilofacial do Hospital Universitário da Universidade Federal de Mato Grosso do Sul e nas clínicas de Cirurgia Bucomaxilofacial da Faculdade de Odontologia da Universidade Federal de Mato Grosso do Sul (UFMS), sendo estes locais próximos ao pronto atendimento médico do Hospital Universitário da UFMS.

\section{Análise dos dados}

A comparação entre os tratamentos (1A ou 1B), em relação ao escore MDAS e da escala de Ramsay foi realizada por meio do teste não paramétrico de Wilcoxon, uma vez que a maior parte das amostras não passaram no teste de normalidade de Shapiro-Wilk. A análise estatística foi realizada por meio do programa estatístico SigmaPlot, versão 12.5, considerando um nível de significância de $5 \%$.

\section{Resultados e Discussão}

Este artigo teve a participação de doze indivíduos com idade entre 18 e 25 anos, sendo média de 21,08 $\pm 0,71$ anos. Em relação ao gênero, $66,7 \%(n=8)$ deles era do sexo feminino e 33,3\% $(n=4)$ do sexo masculino.

No estudo de Labaste et al, 2019, o gênero foi considerado um fator dominante nos escores de ansiedade e dor, sendo as pontuações das mulheres significativamente maiores na resposta de dor. Neste sentido, Sirin et al., 2020, avaliaram a necessidade de sedação em mulheres que tiveram ou não uma experiência odontológica desagradável anterior e avaliaram os possíveis fatores contribuintes. Constataram que quanto mais jovem for a paciente no momento da experiência, pior será o estado de ansiedade, semelhante ao que concluíram nos estudos de Dadalti et al. (2021) e El Hajj et al. (2021).

Dereci et al., 2021, afirmaram em seu estudo que o nível de ansiedade odontológica é maior quando o paciente é submetido a uma exodontia de terceiro molar, do que quando comparada a uma extração de dente convencional.

No presente estudo, para medir o nível de ansiedade dos indivíduos utilizamos a Escala de Ansiedade Odontológica de Corah Modificada (MDAS), assim como realizaram Sghaireen et al (2013); Dao et al. (2014) e López-Jornet et al. (2014). A MDAS possui uma sensibilidade, especificidade, confiabilidade e validade aceitável e é usada em vários ensaios clínicos semelhantes a este com o mesmo propósito. Escolheu-se este questionário para analisar o nível de ansiedade dos voluntários por ser mais apropriado, mais claro e apresentar uma pergunta a mais quando comparada a Escala de Ansiedade Odontológica de Corah (DAS) o que acaba por aumentar sua acurácia (Humphris et al., 1995; Ilguy et al., 2005). A maior parte dos voluntários da amostra foram classificados como extremamente ansiosos de acordo com a pontuação obtida pela MDAS.

$\mathrm{Na}$ análise da ansiedade, com relação a MDAS, não houve diferenças estatisticamente significativas entre os tratamentos 1A e 1B ( $\mathrm{p}=0,742)$, conforme a Tabela 1 . Também não houve diferenças estatisticamente significativas entre a primeira e a segunda cirurgia (primeira cirurgia: $\mathrm{p}=0,084$; segunda cirurgia: $\mathrm{p}=0,359$ ) (Figura 1). Além disso, na primeira consulta a média da MDAS foi de $10,67 \pm 0,88$.

Normalmente, na segunda cirurgia os indivíduos apresentam menor ansiedade quando submetidos à sedação, como encontraram Pereira-Santos et al. (2013) e Paiva-Oliveira et al. (2017), porém neste estudo, os participantes se mantiveram ansiosos em ambas as sessões cirúrgicas, isto pode ser explicado pelo fato de que este ensaio clínico foi composto apenas por participantes ansiosos. 
Tabela 1 - Resultados referentes à avaliação da MDAS, escore na escala de Ramsay, classificação do tratamento.

\begin{tabular}{lrrr}
\hline \multirow{2}{*}{ Variável } & \multicolumn{2}{c}{ Tratamentos } & \multirow{2}{*}{ Valor de p } \\
\hline Escore no MDAS & Valeriana-lúpulo & Midazolam & 0,742 \\
Escala de Ramsay & $11,50 \pm 1,01$ & $11,50 \pm 1,26$ & 0,008 \\
Visão Global do Tratamento & $1,75 \pm 0,13$ & $2,42 \pm 0,15$ & \\
$\quad$ Ótimo & & & \\
Bom & $25,0(3)$ & $41,7(5)$ & \\
Razoável & $41,7(5)$ & $50,0(6)$ & 0,309 \\
Ruim & $25,0(3)$ & $0,0(0)$ & \\
& $8,3(1)$ & $8,3(1)$ & \\
\hline
\end{tabular}

Nota: Os resultados estão apresentados em média terro padrão da média ou em frequência relativa (frequência absoluta). Valor de p no teste de Wilcoxon (variáveis quantitativas) ou no teste do Qui-quadrado (variáveis categóricas). Letras diferentes na linha indicam diferença entre os tratamentos (teste do Qui-quadrado com correção de Bonferroni, p<0,05). Fonte: Autores.

Figura 1 - Gráfico apresentando o escore na MDAS na primeira consulta e em cada uma das cirurgias. Cada coluna representa a média e a barra o erro padrão da média.



Fonte: Autores.

López-Jornet et al. (2014) analisaram a quantidade de ansiedade e medo sentidos antes, imediatamente após e uma semana após a extração dentária. Foram estudados 70 pacientes (35 homens e 35 mulheres) listados para extração dentária sob anestesia local em uma clínica privada especializada em cirurgia oral. Os pacientes foram avaliados em 3 ocasiões consecutivas: imediatamente no pré-operatório, imediatamente no pós-operatório e 7 dias depois. A ansiedade de cada paciente foi medida usando o Inventário de Ansiedade Traço do Estado de Spielberger (versão em espanhol), a Escala de Ansiedade Odontológica de Corah Modificada (MDAS) e o Enquete de Medo Dentário. Concluíram que ansiedade odontológica imediatamente após a extração do dente pode ser influenciada por técnicas operatórias (tipo de anestesia, duração da operação ou posição do dente extraído), mas a ansiedade 7 dias após a extração não parece ter relação.

Em nosso estudo, considerando o número de tubetes anestésicos $(\mathrm{p}=1,000)$ e o tempo de cirurgia $(\mathrm{p}=0,359)$ para cada lado operado não houve diferenças estatisticamente significativas entre os tratamentos realizados. Foram utilizados um volume máximo de 4,5ml de anestésico (2 tubetes e meio) por lado operado, sendo a média de 2,02 ml ou 1,1 tubetes. Em relação ao tempo de cirurgia, a média para o grupo valeriana-lúpulo e para o grupo midazolam foi de 13 minutos, não apresentando diferenças estatisticamente significantes, ratificando a semelhança da amostra. 
Sancak e Akal (2019) avaliaram o efeito de informações verbais e escritas e experiência cirúrgica prévia dos pacientes sobre sua ansiedade antes e após a exodontia do terceiro molar. Foram incluídos 66 pacientes na pesquisa divididos em 3 grupos: o grupo 1 recebeu informações verbais, o grupo 2 recebeu informações por escrito e o grupo 3 teve experiência cirúrgica anterior. O Escala do Estado de Ansiedade (STAI-S), Escala de Medo Dental (DFS), Escala de Ansiedade Odontológica de Corah Modificada (MDAS) e escala visual analógica (VAS) foram usados no pré e pós-operatório para avaliar a ansiedade odontológica. Concluíram que informar os pacientes adequadamente sobre o procedimento de exodontia de terceiros molares reduz a ansiedade pós-operatória. Em nosso estudo todas as informações e orientações pré e pós-operatórias foram passadas aos participantes.

Alguns trabalhos mostram que o nível de ansiedade pré-operatório está associado à sensação de dor pós-operatória nos pacientes operados, e sugerem que para o controle da ansiedade na prática clínica há necessidade de intervenção terapêutica préoperatória com medicação ansiolítica (Van Wijk, Hoogstraten, 2005; Sanikop et al., 2011; Dao et al.,2014; Lopes-Jornet et al., 2014). Em contrapartida, na presente pesquisa não houve relação estatística entre ansiedade pré-operatória (MDAS) com dor pós-operatória (EVA), podendo ser justificado pelo uso da sedação consciente com Midazolam e Valeriana-lúpulo.

Para o nível de sedação, a escala de Ramsay revelou que os participantes tratados com Midazolam tiveram seus escores maiores que o observado no grupo Valeriana-lúpulo (teste de Wilcoxon, $\mathrm{p}=0,008$ ) com diferenças estatisticamente significativas (Tabela 1). O midazolam pode ser utilizado clinicamente seguro em dose de $15 \mathrm{mg}$ ou 7,5 mg (ADA, 2012). Assim, a escolha deste trabalho pelo Midazolam de 15mg possui respaldo científico.

Os efeitos benéficos da sedação pré-operatória facilitam a interação cirurgião e paciente, pois possibilita maior tranquilidade na realização do ato operatório (Either et al., 2006; Donaldson \& Chanpong, 2007).

Cabe salientar que as doses únicas de ansiolíticos no período pré-operatório não possuem a finalidade de tratar o traço de ansiedade dos pacientes que porventura possuam, mas sim apenas o seu estado de ansiedade, no período prévio ao momento cirúrgico, deixando-o tranquilo e relaxado para a cirurgia (Lago-Mendez et al., 2006; López-Jornet et al., 2014).

A Valeriana officinalis, uma planta herbácea da espécie Valerianaceae, tem como uma de suas vantagens o controle a ansiedade, além de improváveis efeitos colaterais quando administrados nas dosagens recomendadas (Pinheiro et al., 2014).

O Humulus Lúpulo age principalmente pela modulação da resposta gabaérgica. Este fitoterápico, teve seu efeito sedativo ratificado após ser utilizado em combinação com a Valeriana officinalis em humanos, ambos agindo de maneira sinérgica como sedativo (Franco et al., 2012).

No estudo de Pinheiro et al. (2014) avaliaram a eficácia da Valeriana $100 \mathrm{mg}$ no controle da ansiedade durante a exodontia de terceiro molar, comparado com grupo placebo. Utilizaram a DAS para esta avaliação. A medicação fitoterápica mostrou-se mais eficaz e com menos alteração da frequência cardíaca do que o grupo placebo.

Corroborando com os nossos resultados, o estudo de Farah et al. (2019), também avaliou a eficácia da valeriana100 mg (no nosso estudo utilizamos uma concentração diferente que foi de $500 \mathrm{mg}$ do extrato fixo de valeriana e 120mg de lúpulo) no controle da ansiedade em exodontia de terceiros molares comparando com o midazolam. Este teve melhor resultado na redução dos parâmetros fisiológicos, porém a valeriana pareceu proporcionar o conforto e relaxamento necessários, sem sedação e sonolência que o midazolam pode causar.

No presente trabalho, o nível de sedação do midazolam obteve maiores escores que a Valeriana com diferenças estatisticamente significativas, consagrando o que a literatura nos reporta sobre a eficácia dos benzodiazepínicos (Cogo et al., 2006; Andrade, 2014; De Morais et al., 2015; Dantas et al., 2017), e o efeito do Midazolam pode ser mais uma vez validado nesta pesquisa, pelo fato de que os participantes ansiosos mesmo permanecendo com estado de ansiedade elevado, tanto na primeira como também nas segundas cirurgias, puderam ter sua ansiedade controlada e atingiram um nível de sedação adequado para realização do procedimento. 
No que tange a visão global do tratamento, não houve diferença estatisticamente significativa entre os tratamentos 1A e 1B, porém na análise descritiva observamos que $41,7 \%$ dos participantes classificaram como ótimo o tratamento com Midazolam contrapondo 25\% desta mesma classificação no tratamento com Valeriana (Tabela 1).

Não foram encontrados na literatura a associação de valeriana-lúpulo na exodontia de terceiros molares inferiores retidos.

Faz-se necessário a elaboração de mais estudos acerca da eficácia e segurança dos fitoterápicos como ansiolítico, assim como trabalhos sobre os efeitos da associação Valeriana-Lúpulo, que são escassos na literatura atual.

\section{Conclusão}

Mesmo sem diferenças significantes nos escores de ansiedade entre a primeira e segunda cirurgia para ambos os tratamentos, a Valeriana pareceu trazer conforto e menos efeitos colaterais que o Midazolam. Ainda assim, novos estudos devem ser elaborados acerca dos efeitos da associação Valeriana-Lúpulo para controle da ansiedade odontológica.

\section{Agradecimentos}

O presente trabalho foi realizado com apoio da Fundação Universidade Federal de Mato Grosso do Sul -UFMS/MEC -Brasil.

\section{Referências}

American Dental Association. (1999). American Dental Association Guidelines for the Use of Conscious Sedation, Deep Sedation and General Anesthesia for Dentists. ADA.

American Dental Association. (2012). Guidelines for the use of sedation and general anesthesia by dentists, 2007. American Dental Association.

Andrade, E. D. (2014). Terapêutica medicamentosa em odontologia. Artes Médicas Editora.

Armfield, J. M., \& Heaton, L. J. (2013). Management of fear and anxiety in the dental clinic: a review. Australian Dental Journal. 58(4), 390-407.

Braga, A. D. F. D. A, D’Ottaviano, L. H., Braga, F. S. D. S, \& Morais, S. S. (2010). Extração de Terceiros Molares Retidos sob Anestesia Local. Avaliação de Ansiedade, Dor, Alterações Hemodinâmicas e Respiratórias. Revista da Faculdade de Odontologia. 51(2), 9-14.

Cogo, K., Bergamaschi, C. C., Yatsuda, R., Volpato, M. C., \& Andrade, E. D. (2006). Sedação Consciente Com Benzodiazepínicos em Odontologia. Revista de Odontologia da Universidade de São Paulo. 18(2), 181-8.

Dadalti, M. T. S., Cunha, A. J. L., Souza, T. G. D., Silva, B. A., Luiz, R. R., \& Risso, P. A. (2021). Anxiety about dental treatment - a gender issue. Acta Odontologica Latinoamericana. 34 (1), 195-200.

Dantas, L. P., de Oliveira-Ribeiro, A., de Almeida-Souza, L.M., \& Groppo, F. C. (2017). Effects of passiflora incarnata and midazolam for control of anxiety in patients undergoing dental extraction. Medicina Oral, Patologia Oral, Cirugia Bucal. 22(1), 95-101.

Dao, J., Zhang, J., Song, G., \& Xin, Q. (2014). Effect of preoperative anxiety level on postoperative pain sensation in patients receiving implant denture for partial edentulism. Nan Fang Yi Ke Da Xue Xue Bao, 34(4), 528-31.

De Morais, H. H., Barbalho, J. C., de Holanda Vasconcellos, R. J., Landim, F. S., da Costa Araujo, F. A., \& de Souza Dias, T. G. (2015). Comparative study of hemodynamic changes caused by diazepam and midazolam during third molar surgery: a randomized controlled trial. Oral and Maxillofacial Surgery. 19(3), 267-73.

Dereci, O., Saruhan, N., \& Tekin, G. (2021). The Comparison of Dental Anxiety between Patients Treated with Impacted Third Molar Surgery and Conventional Dental Extraction. Biomed Research International. doi.org/10.1155/2021/7492852.

Dhuvad, J. M., Kshisagar, R. A., \& Dhuvad, M. M. (2014). Comparative evaluation of vital parameters during third molar surgery under local anaesthesia with and without sedative agents. Journal of Clinical and Diagnostic Research. 8(12), 57-60.

Donaldson, M., Gizzarelli, G., \& Chanpong, B. (2007). Oral sedation: a primer on anxiolysis for the adult patient. Anesthesia Progress. 54(3),118-28.

Either, S., Wichmann, M., Paulsen, A., \& Holst, S. (2006). Dental anxiety - an epidemiological study on its clinical correlation and effects on oral health. Journal of Oral Rehabilitation. 22(8), 588-593.

El Hajj, H. K., Fares, Y., \& Abou-Abbas, L. (2021). Assessment of dental anxiety and dental phobia among adults in Lebanon. BMC Oral Health. 21(1), 48. 
Farah, G. J. 2010. Como controlar a ansiedade do paciente frente a procedimentos odontológicos? Revista Dental Press de Periodontia e Implantologia. 4(2), 33-8.

Farah, G. J., Ferreira, G. Z., Danieletto-Zanna, C. F., \& Luppi, C. R. (2019). Assessment of Valeriana officinalis 1. (Valerian) for Conscious Sedation of Patients During the Extraction of Impacted Mandibular Third Molars: A Randomized, Split-Mouth, Double-Blind, Crossover Study. Journal of Oral and Maxillofacial Surgery. 77(9), 1796.e1-1796.

Franco, L., Sánchez, C., Bravo, R., Rodriguez, A., Barriga, C., \& Cubero, J. C. (2012). The sedative effects of hops (Humulus lupulus), a component of beer, on the activity/rest rhythm. Acta Physiologica Hungarica. 99(2), 133-39.

Guzeldemir, E., Toygar, H. U., \& Cilasun, U. (2008). Pain perception and anxiety during scaling in periodontally healthy subjects. Journal of Periodontology. 79(12), 2247-55.

Humphris, G. M., Morrison, T., \& Lindsay, S. J. E. (1995). The Modified Dental Anxiety Scale: validation and United Kingdom norms. Community Dental Health. 12, 143-50.

Ilguy, D., Ilguy, M., Dincer, S., \& Bayirli, G. (2005). Reliability and validity of the modified dental anxiety scale in turkish patients. Journal of International Medical Research. 33, 252-59.

Joshi, S., Ansari, A. S. A., Mazumdar, S., \& Ansari, S. (2016). A comparative study to assess the effect of oral alprazolam as premedication on vital parameters of patients during surgical removal of impacted mandibular third molars. Contemporary Clinical Dentistry. 7(4), 464-68.

Kinrys, G., Coleman, E., \& Rothstein, E. 2009. Natural remedies for anxiety disorders: potential use and clinical applications. Depression and Anxiety. 26, 25965 .

Labaste, F., Ferré, F., Combelles, H., Rey, V., Foissac, J. C., Senechal, A., Conil, J. M., \& Minville, V. (2019). Validation of a visual analogue scale for the evaluation of the postoperative anxiety: A prospective observational study. Nursing Open. 6(4), 1323-30.

Lago-Mendez, L., Diniz-freitas, M., Senra-Rivera, C., Seoane-Pesqueira, G., Gandara-Rey, J. M., \& Garcia, A. (2006). Dental anxiety before removal of a third molar and association with general trait anxiety. Journal of Oral and Maxillofacial Surgery. 64(9), 1404-8.

López-Jornet, P., Camacho-Alonso, F., \& Sanchez-Siles, M. (2014). Assessment of general pre and postoperative anxiety in patients undergoing tooth extraction: a prospective study. British Journal of Oral and Maxillofacial Surgery. 52(1), 18-23.

Ong, C. K. S., Lirk, P., Seymour, R. A., \& Jenkins, B. J. (2005). The efficacy of preemptive analgesia for acute postoperative pain management: a meta-analysis. Anesthesia \& Analgesia. 100(3), 757-73.

Paiva-Oliveira, J. G. (2017). Ensaio clínico randomizado: analgesia preemptiva na associação ibuprofeno e dexametasona sob sedação consciente mínima e avaliação da ansiedade e sinais vitais em exodontias de terceiros molares inferiores [teses]. Campo Grande: Universidade Federal de Mato Grosso do Sul.

Pereira-Santos, D., Brêda-Júnior, M. A., Ferraz, E. P., Crippa, G. E., de Oliveira, F. S., \& da Rocha-Barros, V. M. (2013). Study comparing midazolam and nitrous oxide in dental anxiety control. The Journal of Craniofacial Surgery. 24(5), 1636-9.

Pinheiro, M. L. S., Alcântara, C. E. P., Moraes, M., \& Andrade, E. D. (2014). Valeriana officinalis L. for conscious sedation of patients submitted to impacted lower third molar surgery: A randomized, double-blind, placebo-controlled split-mouth study. Journal of Pharmacy and Bioallied Sciences. 6(2), 109-14.

Possobon, R. D. F., Camillo, C. K., Alves de Moraes, A. B., \& Costa, J. R. (2007). O tratamento odontológico como gerador de ansiedade. Psicologia em Estudo, 12(3), 609-16.

Sanikop, S., Agrawal. P. \& Patil, S. (2011). Relationship between dental anxiety and pain perception during scaling. Journal of Oral Science. 53(3), 341-8.

Sancak, K. T., \& Akal, U. K. (2019). Efeito da informação verbal e escrita e da experiência cirúrgica anterior na ansiedade durante a extração do terceiro molar. Journal of Oral and Maxillofacial Surgery. 77 (9), 1769.e11769.

Sghaireen, M. G., Zwiri, A. M., Alzoubi, I. A., Qodceih, S. M., \& Al-Omiri, M. K. (2013). Anxiety due to Dental Treatment and Procedures among University Students and Its Correlation with Their Gender and Field of Study. International Journal of Dentistry. 647436.

Sirin, Y., Yildrimturk, S., \& Ay, N. (2020). Do state-trait anxiety and previous unpleasant dental experiences predict the need for sedation in women having third molar surgery? Bristish Journal of Oral and Maxillofacial Surgery. 58, 530-34.

Van Wijk, A. J., \& Hoogstraten, J. (2005). Experience with dental pain and fear of dental pain. Journal of Dental Research. 84, 947-50. 